(C) 2017 IEEE. Personal use of this material is permitted. Permission from IEEE must be obtained for all other uses, in any current or future media, including reprinting/republishing this material for advertising or promotional purposes, creating new collective works, for resale or redistribution to servers or lists, or reuse of any copyrighted component of this work in other works. 


\title{
METHODS FOR DETERMINING CAPACITY IN AN ALTERNATE MODE OF OPTICAL DATA STORAGE
}

\author{
Kelly Goss *, Mike Potter *, Richard W. Ziolkowski **, Mark A. Neifeld ** \\ *University of Calgary, Calgary, Canada \\ Email:kgoss@ieee.org \\ **University of Arizona, Tucson, USA \\ Email: ziolkowski@ece.arizona.edu
}

\section{INTRODUCTION}

Current data storage techniques are based on magnetic and optical technologies such as hard drives and compact discs (CDs) respectively. Research in magnetic technologies has achieved data densities of 100 Gbits/in $^{2} \quad(\sim 15$ Gbits $\left./ \mathrm{cm}^{2}\right)[1]$. With the more recent discovery of optical data storage research has greatly improved data densities with implementations such as the DVD that consists of multiple layers of CDs and Blu-Ray technology that uses a shorter wavelength optical source to decrease the feature size and increase data density up to $2 \mathrm{Gbits} / \mathrm{cm}^{2}$. Although research continues to improve these technologies, both magnetic and optical data storage are limited in their readout speeds due to their serial readout nature.

An alternate optical data storage technique is proposed that aims to surpass the data densities achieved by magnetic storage and allow for bits to be read in parallel. The system will store data in sub-wavelength surface relief profiles that will be read using near-field optical transmitted power measurements. This paper begins by describing how the proposed system will store and retrieve data. The focus of current research is then discussed which investigates an appropriate method to simulate and estimate the data storage capacity of the system so that future work can be aimed at optimizing the parameters of the system.

\section{PROPOSED SYSTEM}

Memory is simply any system that has a large number of distinguishable states. This proposed system will create multiple states within sub-wavelength structures and therefore achieve multiple bits per wavelength. These structures will consist of two materials referred to as the base and fill. Between a homogeneous layer of fill and base, a feature layer will allow for multiple states to be created by different compositions of fill and base material. To distinguish different states, the feature layer is divided into vertical subsections. Each subsection can consist of either the fill or base material. By assigning different combinations of material to different subsections, different states are created. Figure 1 is an example of a system that has 5 features which amounts to $2^{5}=32$ physical states.

To retrieve data it is necessary to be able to distinguish between the different physical states of the system. The proposed system aims to accomplish this by reading the optical power transmitted through the structures, as shown in Figure 1. These multiple power measurements will create a vector of measurements, $\mathrm{P}=\left(\mathrm{P}_{1}, \mathrm{P}_{2}, \ldots, \mathrm{P}_{\mathrm{n}}\right)$ where $\mathrm{n}$ is the number of sensors. The different possible compositions of the feature layer will cause a different interaction with the optical source, which will lead to a different power vector and the identification of different states.

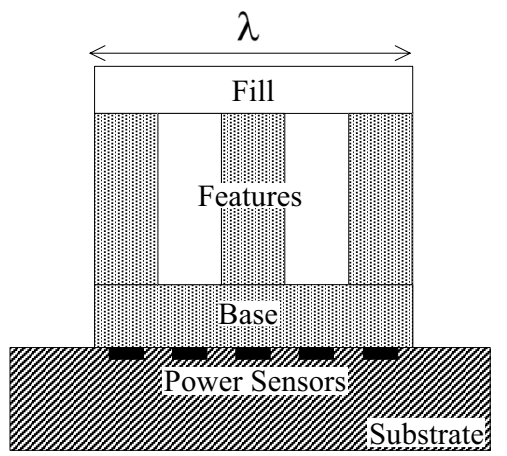

Figure 1 - Nano-scale cells with base, feature and fill layers

It is important to note that Figure 1 only illustrates one cell that corresponds to one set of bits. For this system to store a large amount of data and at a high density, it is necessary to place these cells as close to each other as possible. Figure 2 illustrates one portion of a system, which consists of three structures.
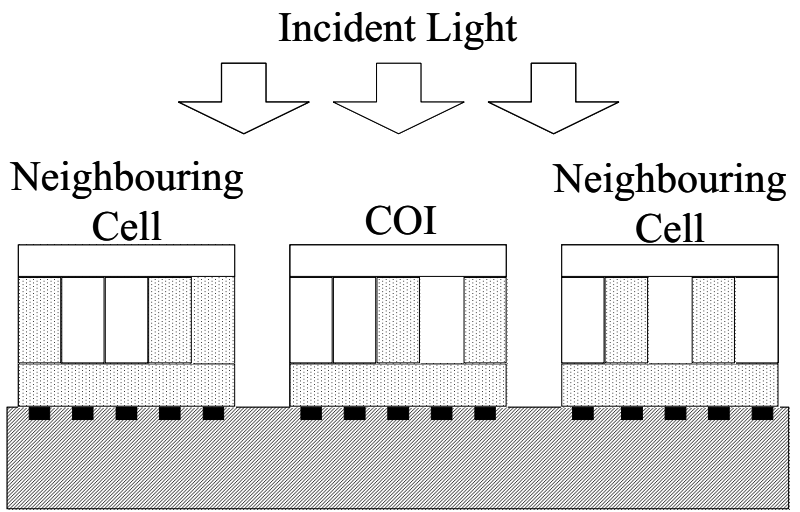

Figure 2- System Layout

The cell of interest (COI), as labelled in Figure 2, is the structure which we are trying to identify. The other two structures are referred to as neighbouring cells. All cells have power sensors, allowing for simultaneous 
measurements for all the illuminated cells and therefore readout of data in parallel.

However, neighbouring cells will also interact with the optical source and produce interference, which will affect the power measurements of the COI. This introduces uncertainty in the measure of the COI since different neighbours will effect the measurements differently. To illustrate this we will examine a simplified system with only two power measurements. The power vector would then consist of two values, $\mathrm{P}=\left(\mathrm{P}_{1}, \mathrm{P}_{2}\right)$, and we can think of this as a coordinate in a plane. Figure 3 plots one power measurement, $P_{1}$, versus another, $P_{2}$ for three cells of interest.

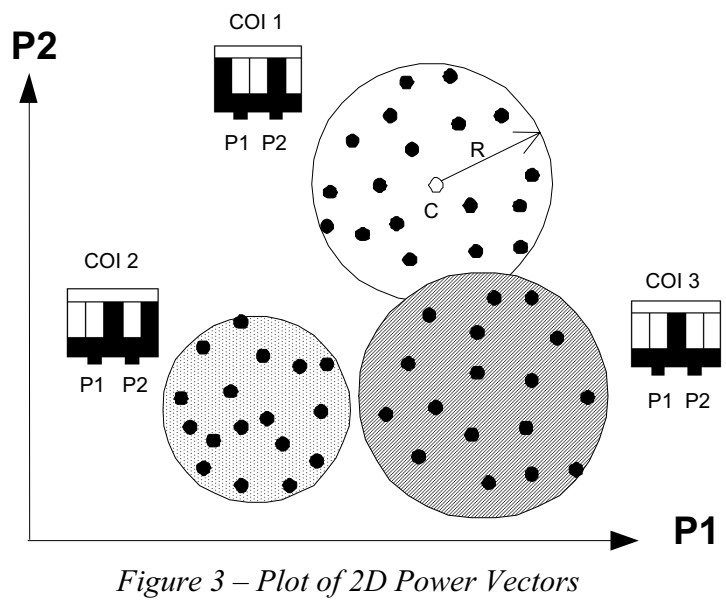

Each COI has a set of power vectors associated with it, which correspond to the different power measurements resulting from different neighbouring cells. The region these sets encompass are defined by a centre vector and a scalar radial distance from the centre to the furthest point as shown for COI 1 in Figure 3. We refer to these sets as power vector clouds. For a COI to be distinguishable, it is necessary that its set of power vectors be unique from all other COIs. In Figure 3, COI 2 is distinguishable since it does not overlap with COI 1 and COI 3. However, COI 1 and COI 3 are not distinguishable since their power vector clouds overlap.

Obviously the best system will have maximum distinguishability of COIs. To investigate how different parameters of the system affect the optical interactions a method is required to simulate different systems and to analyze the results to approximate capacity. This current focus of our research is now discussed in the following sections.

\section{SIMULATIONS}

To simulate the system a two-dimensional Finite Difference Time Domain (FDTD) algorithm is used which segments the system space and solves discrete Maxwell equations for the electric and magnetic fields at each segment [2]. FDTD is used for this system because of its flexibility with geometric structures. A high resolution of $\lambda / 100$ is used to discretize the system. This yields a $450 \mathrm{x}$ 522 computational domain. An 11 layer two-time derivative Lorentz material (TDLM) [3] formulation is used for absorbing boundary conditions. The optical source is a pulsed Gaussian beam normally incident to the structure with a wavelength of $500 \mathrm{~nm}$. The simulation is iterated over approximately 20 cycles of the source. The outputs of the simulations are the electric and magnetic fields observed at the sensor locations, which are used to determine the power measurements.

The initial simulations were performed with only the cell of interest to determine which set of parameters allowed for the largest separation between COI power vectors. Two promising candidates, one based on silicon and the other on polymer, were identified. The dimensions of these structures are shown in Figures 4 and 5.
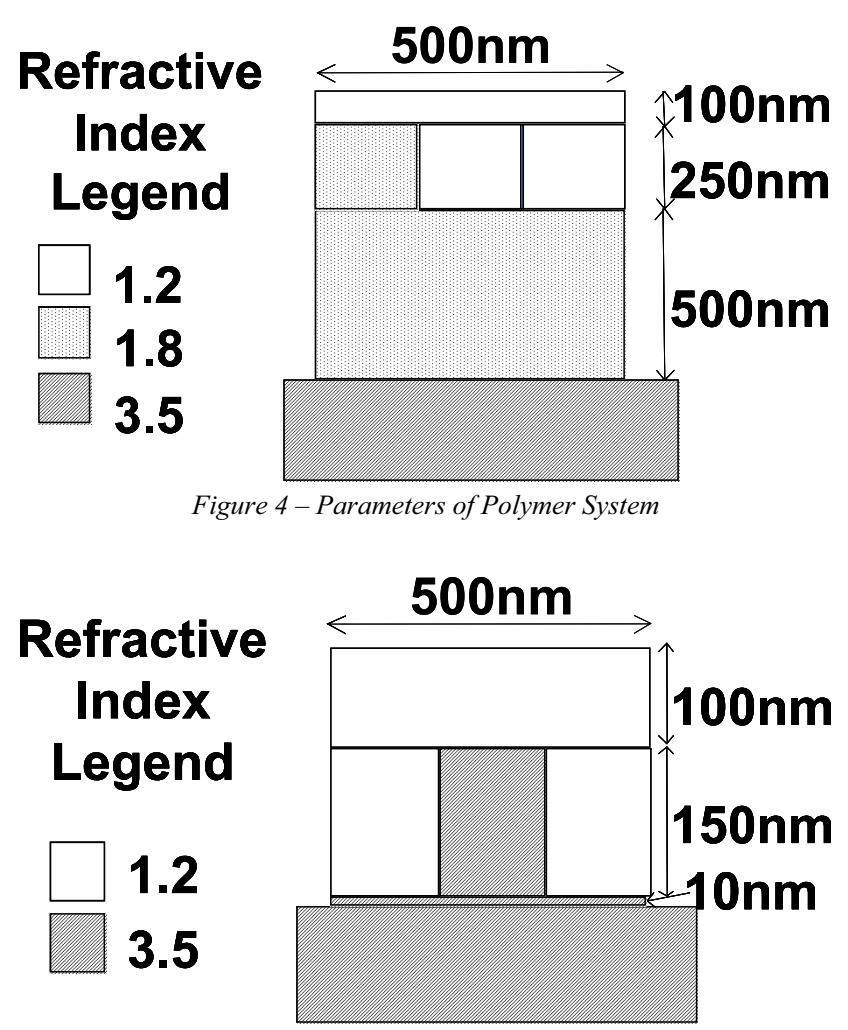

Figure 5 - Parameters of Silicon System

With promising cells of interest identified, it was next necessary to determine the interference introduced by the neighbours. Therefore for every system, each COI needs to be simulated with every possible combination of neighbours. As previously indicated, a COI with 5 features has $2^{5}=32$ states. There are also the same number of possible configurations for each neighbouring cell which results in $32^{3}=32768$ total simulations. This results in an estimated 3 months of simulation per system. Since parameterization of this system will require several systems to be simulated this is not a practical simulation method. 


\section{INITIAL ESTIMATES OF CAPACITY}

To gain an initial estimate of the capacity of the system, simulations were performed with 5 features but only 3 neighbour configurations. The three neighbouring configurations in Figure 6 were chosen in attempt to cover the range of interference from the neighbours.

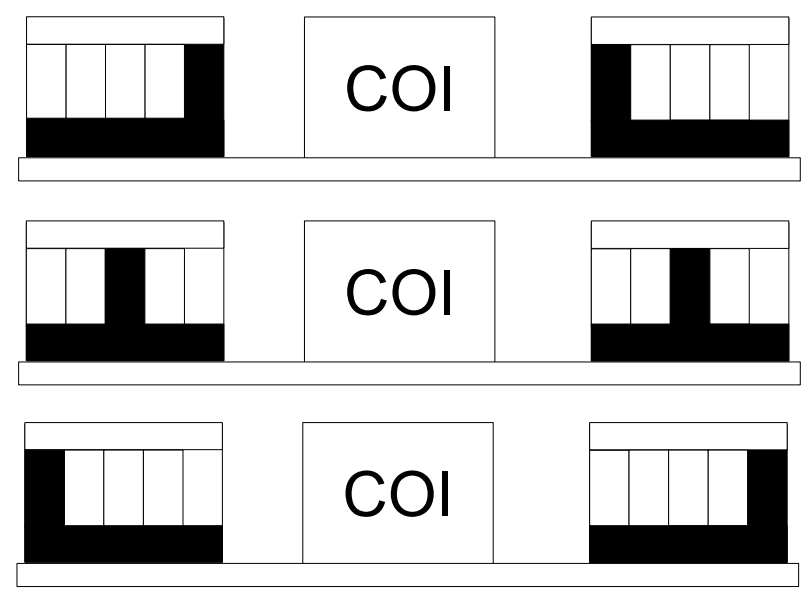

Figure 6-Neighbour configurations used for initial capacity analysis

With these three neighbouring configurations, three power vectors were attained for each COI. The centre was calculated as the mean and the radius of the power vector clouds was approximated by the furthest distance between any of the three power vectors. Simulations were performed on both the silicon and polymer based systems. For both the silicon and polymer system not all 32 states were distinguishable. However at least 16 states were distinguishable in both systems which corresponds to 4 bits per structure which can still be used to store data. Assuming that the same density can be achieved in the perpendicular direction, this system would attain a data density of $5 \mathrm{Gbits} / \mathrm{cm}^{2}$.

\section{BETTER ESTIMATION METHODS}

Although these are some promising initial results, it is necessary to determine how closely the three neighbour configurations approximate the centre and radius of the power vector clouds. To do this, simulations were performed on a system with only three features. For a system with only three features, there are $2^{3}=8$ COIs and therefore $8^{3}=512$ simulations. Although this is still a large amount of simulations, it is significantly smaller than the 5 feature system. This enables us to simulate all the neighbour configurations for a system in a reasonable amount of time. With this full set of neighbours and therefore a full set of power vectors, the actual radius can be calculated and can be used to determine the error when approximating the radius with only a fraction of neighbours.

The three feature systems were simulated for both the polymer and silicon. Using the three neighbour configurations shown in Figure 6 the radii for each COI were estimated for the three feature system. Since the full set of neighbours were simulated, the actual radii of each COI power vector cloud is known and used to calculate the error in the three neighbour estimation. The average error in the radii estimations was $52 \%$ and $2 \%$ for the center estimation. The error in the centre is small enough to be satisfactory. Although the error in the radius is large, it is important to note that all of the radius estimations were larger than the actual radius.

To improve the accuracy of the radius estimation, three different methods have been investigated. These three methods are based on estimating the radius from a subset of neighbours in the following way:

1) The radius is estimated as the largest distance between any two points.

2) The radius is estimated as the distance from the furthest point to the center.

3) The radius is estimated in the same was as Method 2; however, one of the neighbouring configurations in the subset is one of the ten furthest points from the center. The other neighbouring configurations are chosen randomly.

Method 1 is the method that was used to estimate the radii with only the three configurations whereas method 2 is the method used to calculate the actual radii with the full set of neighbours. Method 3 is proposed with the assumption that for a given COI the neighbours that contribute the most interference will be the same for different systems.

The algorithm for method 3 involves an initial analysis, which for each COI using the full set of neighbours determines the ten vectors that are the furthest away from the centre. Then by randomly choosing one of these ten vectors and randomly choosing the rest from the full set of vectors, estimates the radius using method 2 .

To compare these methods, they were each repeated 500 times with a random set of neighbours. The fraction of the set of neighbours was increased from 3 out of 64 neighbours to the full set of 64 out of 64 neighbours. The error was calculated from the known radius determined by using method 2 with all the neighbours, ie. fraction $=1$. Figures 7 and 8 show the RMS percent error in the radius estimation for all three methods versus the fraction of neighbours for the polymer and silicon system respectively.

With the full set of vectors we would expect the distance between the furthest two points to be much larger than the radius of the circle and hence with method 1 the error in the radius estimation increases with larger sets of vectors. We would expect the error in the radius to decrease with method 2 since this is the technique used to calculate the actual radius with a full set. And similarly with method 3 we would expect a similar trend to method 2 but with a smaller error since one of the furthest ten points is always included in the set. 


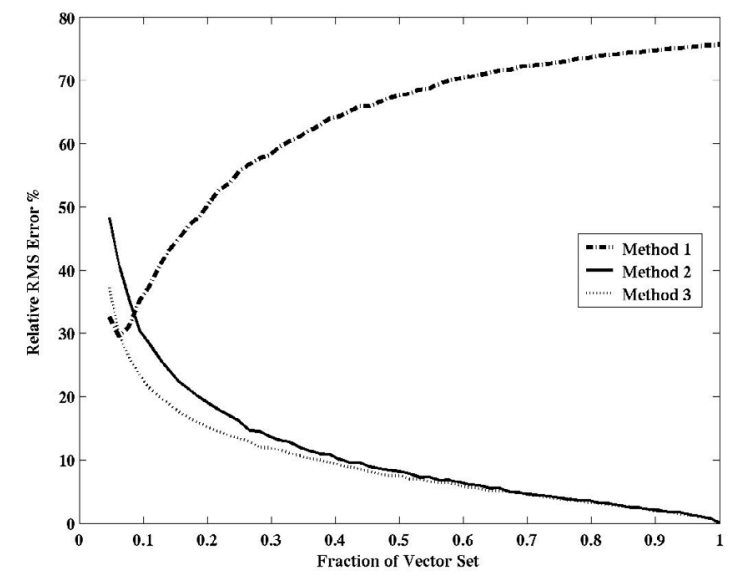

Figure 7 - RMS Error in radius estimation averaged over COIs for the polymer system.

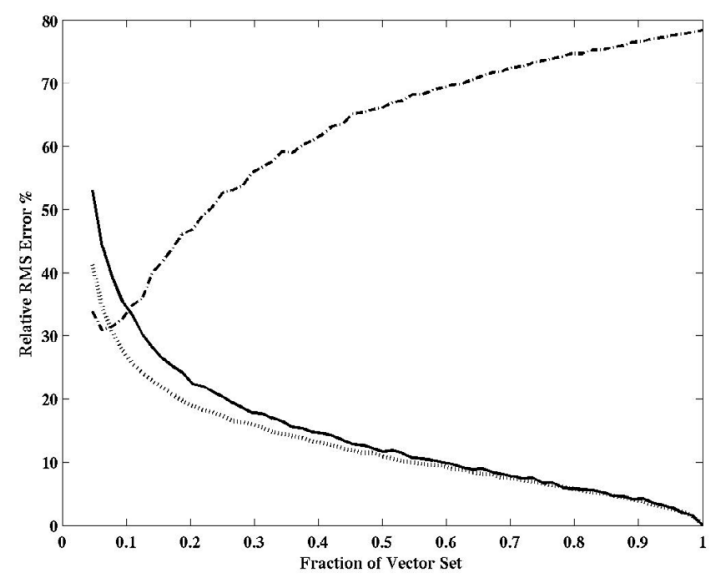

Figure 8-RMS Error of radius estimation averaged over COIS for the silicon system.

Using these results from the 3-feature system simulations we expect that when simulating the 5-feature system that we can get a better estimation of the radius with judicious choice of neighbours. Currently simulations are being performed with three features to verify and that the neighbours that introduce the most interference are the same for different systems.

In addition since we know an upper bound on the error at a certain fraction, then we can at least get a worse-case scenario estimation of the capacity by adjusting the error accordingly. For example, if we know the upper bound of the error is $50 \%$, then if we multiply the estimated radius by 1.5 before determining capacity, then we can get a conservative estimate on capacity.

Referring back to the initial capacity estimations we can now use these results to make a conservative estimate on the capacity. With the three neighbours out of 1024 for the 5 feature system, this corresponds to a fraction of $\mathrm{f}=0.003$. From our error versus fraction graphs we are limited to a fraction of $3 / 64 \approx 0.05$; however, if we estimate the error to be $50 \%$ we can determine that with the polymer system can no longer distinguish 16 states and therefore the data density drops. However, with the silicon system 16 states are still distinguishable so the original density estimates still hold with our conservative estimate.

\section{CONCLUSION}

An alternate mode of optical data storage has been proposed. This system aims to surpass magnetic data densities and allow for parallel readout. The system is based on the identification of inhomogeneous nano-scaled structures based on transmitted optical power measurements. Simulations are performed using FDTD and initial capacity estimates are $5 \mathrm{Gbits} / \mathrm{cm}^{2}$.

The problem with simulations arise from the fact that with 5 features per wavelength this results in over 32000 simulations per system. The current focus of the work is to determine how better to calculate capacity using only a subset of simulations. Some initial methods have been proposed and the error in these methods has been quantified for a 3 feature system. This data can now be used to better estimate the capacity of a system with only a subset of simulations.

\section{FUTURE WORK}

With an initial estimate of capacity and better estimation methods, the next steps focus on increasing the capacity of the system. Some possible parameters to explore include different polarization and source wavelength. These parameters and others will provide additional information which could be useful for distinguishing different states. Another evident path to explore is simulating the system in three dimensions to determine the available capacity in the extra dimension.

\section{REFERENCES}

[1] Z.Zhang et al., "Magnetic Recording Demonstration Over 100Gb/in'," IEEE Transactions on Magnetics, vol. 38, pp.1861-1866, September 2002.

[2] A. Taflove and S.C. Hagness, Computational Electrodynamics: The Finite-Difference Time-Domain Method. Boston: Artech House, 2000.

[3] D.C. Wittwer and R.W. Ziolkowski, "Two timederivative Lorentz material (2TDLM) formulation of a Maxwellian absorbing layer matched to a lossy medium," IEEE Transactions on Antennas and Propagation, vol. 48, pp192-199, Feb 2000. 